\title{
PLANTAS MEDICINAIS: INDICAÇÃO DE RAIZEIROS PARA O TRATAMENTO DE FERIDAS
}

\author{
Medicinal plants: traditional healers' indications for the \\ treatment of wounds
}

\author{
Plantas medicinales: indicación de herboristas para el \\ tratamiento de heridas
}

Artigo Original

\section{RESUMO}

Objetivo: Analisar as indicações e o conhecimento de raizeiros quanto ao uso de plantas medicinais para o tratamento de feridas. Métodos: Estudo quantitativo, de caráter descritivo, realizado com 32 raizeiros do município de Campina Grande - PB, no período de novembro de 2012 a março de 2013. Utilizou-se um questionário estruturado, contendo 21 questões fechadas e abertas, referentes a variáveis sociodemográficas, informações sobre as espécies vegetais indicadas para o tratamento de feridas, aquisição de conhecimentos sobre o assunto e comercialização das plantas. Realizou-se análise descritiva dos dados com cálculo de frequências absolutas e relativas. Resultados: Dos 40 comerciantes de plantas convidados a participar da pesquisa, $32(75 \%)$ consentiram a entrevista, dos quais $75 \%(n=24)$ do sexo feminino, $50 \%(\mathrm{n}=16)$ possuíam 60 anos ou mais, $25 \%(\mathrm{n}=8)$ residiam na zona rural, e apenas $18,75 \%(n=6)$ cultivavam as ervas que comercializavam. Neste ramo, 87,5\% $(n=28)$ trabalhavam há mais de cinco anos, sendo a transmissão familiar através das gerações a forma mais comum de aquisição de conhecimentos $(87,5 \%$; $n=28)$. Um total de 18 plantas foi citado pelos raizeiros, onde o cajueiro roxo foi o mais indicado $(87,5 \% ; n=28)$, seguido pelo barbatimão $(81,25 \%$; $n=26)$ e pela quixaba $(50 \% ; n=16)$. Conclusão: Este estudo deixa em aberta a questão sobre o nível de conhecimento popular de raizeiros investigados acerca do uso de plantas medicinais no tratamento de feridas, visto que o mesmo advém de fonte familiar sem cientificidade, com imprecisa indicação terapêutica.

Descritores: Plantas Medicinais; Ferimentos e Lesões; Cicatrização; Etnofarmacologia.

\section{ABSTRACT}

Objective: To analyze the indications and knowledge of traditional healers on the use of medicinal plants for the treatment of wounds. Methods: Quantitative descriptive study conducted with 32 traditional healers from the municipality of Campina Grande-Paraiba, from November 2012 to March 2013. We used a structured questionnaire with 21 closedand open-ended questions on sociodemographic variables, information on the plant species indicated for the treatment of wounds, acquisition of knowledge on the issue and sale of plants. Data underwent descriptive analysis using absolute and relative frequencies. Results: Of the 40 medicinal plants dealers invited to participate in the study, 32 (75\%) agreed to answer the interview; of these, $75 \%(n=24)$ were women, $50 \%(n=16)$ were 60 years old or older, $25 \%(n=8)$ lived in rural areas, and only $18.75 \%(n=6)$ grew the herbs they traded. In all, $87.5 \%(n=28)$ of the respondents worked for over five years in this sector, with the family transmission across generations as the most common form of knowledge acquisition $(87.5 \% ; n=28)$. In all, 18 plants were cited by the traditional healers, with the purple cashew tree as the most indicated $(87.5 \% ; n=28)$, followed by barbatimão $(81.25 \% ; n=26)$ and quixaba (50\%; $n=16)$. Conclusion: The present study leaves question about the level of popular knowledge of traditional healers on the use of medicinal plants for the treatment of wounds unanswered given that it comes from a family source without scientific evidence and imprecise therapeutic indication.

Descriptors: Medicinal Plants; Wounds and Injuries; Wound Healing; Ethnopharmacology.

Recebido em: 11/03/2016 Revisado em: 01/04/2016 Aceito em: 30/05/2016

1) Universidade Estadual da Paraíba UEPB - Campina Grande (PB) - Brasil 


\section{RESUMEN}

Objetivo: Analizar las indicaciones y el conocimiento de herboristas sobre el uso de plantas medicinales para el tratamiento de heridas. Métodos: Estudio cuantitativo, de carácter descriptivo realizado con 32 herboristas del municipio de Campina Grande - PB en el periodo entre noviembre de 2012 y marzo de 2013. Se utilizó un cuestionario estructurado con 21 cuestiones tipo test y de desarrollar referentes a las variables socio demográficas, informaciones de las especies de vegetales indicadas para el tratamiento de heridas, adquisición de conocimientos sobre el asunto y el comercio de las plantas. Se realizó un análisis descriptivo de los datos con cálculo de frecuencias absolutas y relativas. Resultados: De los 40 comerciantes de plantas invitados a participar de la investigación, 32 (75\%) permitieron la entrevista de los cuales el 75\% $(n=24)$ era del sexo femenino, el 50\% $(n=16)$ tenía 60 años ó más, el $25 \%(n=8)$ vivía en la zona rural y solamente el $18,75 \%(n=6)$ cultivaba las hierbas que comercializaban. En esta especialidad, el $87,5 \%(n=28)$ trabajaba hace más de cinco años y la transmisión familiar entre generaciones fue la forma más común de adquirir conocimientos $(87,5 \%$; $n=28)$. Dieciocho plantas fueron citadas por los herboristas de las cuales el "cajueiro" morado fue el más indicado (87,5\%; $n=28)$ seguido del "barbatimão" (81,25\%; $n=26)$ y la "quixaba" (50\%; $n=16)$. Conclusión: Este estudio abre una cuestión sobre el nivel de conocimiento popular de los herboristas investigados sobre el uso de las plantas medicinales para el tratamiento de heridas una vez que el mismo viene de la fuente familiar sin cientificidad y con indicación terapéutica vaga.

Descriptores: Plantas Medicinales; Heridas y Traumatismos; Cicatrización de Heridas; Etnofarmacología.

\section{INTRODUÇÃO}

Desde os primórdios da civilização, o homem acumulou informações e experiências sobre a utilização de recursos naturais para o tratamento de doenças e sintomas ${ }^{(1)}$. Neste contexto, o uso de espécies vegetais foi uma prática que ultrapassou todas as barreiras do processo evolutivo, sendo um recurso terapêutico amplamente utilizado até nos dias atuais, em várias partes do mundo e com grande significado em algumas regiões mais carentes do $\operatorname{Brasil}^{(2,3)}$.

A Organização Mundial da Saúde (OMS) estima que $85 \%$ da população mundial fazem uso de plantas medicinais para os cuidados com a saúde, e $80 \%$ das pessoas dos países em desenvolvimento dependem de práticas tradicionais e ou complementares para manutenção ou recuperação da saúde, a exemplo das práticas populares de cuidados com feridas $^{(4)}$.

Esta situação pode ser influenciada pela questão econômica (alto custo dos medicamentos alopáticos, dificuldades no acesso aos serviços de assistência médica e dispensação de fármacos) ou pelo simples fato de ser uma tendência atual de substituição dos medicamentos sintéticos (medicina convencional) para a utilização de plantas medicinais (medicina alternativa) $)^{(1,5)}$.

As plantas medicinais possuem princípios ativos, os quais são produzidos durante o seu metabolismo, que lhes conferem ação terapêutica ${ }^{(6)}$. São vegetais utilizados como remédios caseiros, com finalidade de tratar doenças, podendo até levar a cura ${ }^{(7)}$.

No Brasil, é notório que a miscigenação das culturas indígenas, africanas e europeias foram grandes influenciadoras no que concerne a utilização de ervas nos tratamentos de doenças e feridas ${ }^{(8)}$.

No processo de cicatrização de feridas, desde a préhistória, os produtos de origem vegetal eram utilizados de forma tópica, como cataplasmas, com o intuito de estancar hemorragias e favorecer a cicatrização, ou eram ingeridas soluções para atuar de forma sistêmica ${ }^{(9)}$.

De modo geral, as plantas são indicadas e administradas após um ferimento cutâneo, por promoverem a coagulação do sangue, combaterem infecções e acelerarem o processo de cicatrização, que consiste em uma sequência de eventos formada por três fases evolutivas sobrepostas: fase inicial ou inflamatória, fase proliferativa ou de fibroplasia, e fase de remodelação ou maturação. Além de propiciar o rápido fechamento da lesão, alcançar uma cicatriz funcional e esteticamente satisfatória, as ervas são consideradas mais econômicas e acessíveis que os fármacos sintéticos ${ }^{(10,11)}$.

Uma figura que se destaca na indicação, comercialização e manipulação de plantas medicinais é o raizeiro (herbolário ou ervanário, como também são conhecidos os comerciantes e conhecedores de plantas medicinais ${ }^{(12)}$, reconhecido como agente possuidor de uma cultura tradicional e responsável por realizar o comércio destes produtos em feiras livres, mercados populares e em entrepostos (pontos de vendas em residências ou vendedores de rua) $)^{(13)}$.

Diante desse contexto, pode-se observar que as plantas com propriedades medicinais e o profissional raizeiro são de extrema importância para o tratamento alternativo de doenças, assim como de ferimentos. Desta forma, deve-se reconhecer que este é um campo de interação de práticas e saberes, e para que todas as suas potencialidades sejam aproveitadas ao máximo na promoção à saúde é necessário que haja a valorização dos recursos culturais, das riquezas naturais e das relações interpessoais (entre os usuários, os profissionais da área da saúde e os raizeiros), no intuito de desenvolver uma visão crítica na população e promover a socialização da pesquisa científica referente ao uso de plantas na atenção primária a saúde e no setor familiar ${ }^{(14,15)}$.

Diante do exposto, o objetivo deste estudo consistiu em analisar as indicações e o conhecimento de raizeiros quanto ao uso de plantas medicinais para o tratamento de feridas. 


\section{MÉTODOS}

Trata-se de um estudo do tipo descritivo, de natureza quantitativa, realizado com raizeiros de feiras livres do município de Campina Grande-PB, no período de novembro de 2012 a março de 2013.

Como critérios de inclusão na pesquisa, adotaramse: ser maior de 18 anos, comerciante e conhecedor de plantas medicinais utilizadas no tratamento de feridas, estar trabalhando no momento da coleta de dados e aceitar participar voluntariamente da pesquisa. Excluíramse os indivíduos que não se enquadraram nos critérios pré-estabelecidos. Após a explicação dos objetivos e metodologia da pesquisa, os raizeiros foram convidados a participar da pesquisa. Todos os que concordaram em participar assinaram o Termo de Consentimento Livre e Esclarecido (TCLE), assegurando aos voluntários da pesquisa sua privacidade e a liberdade de participar ou desistir a qualquer momento do estudo.

O presente estudo possui uma amostra do tipo não probabilística por acessibilidade, visto que buscou entrevistar todos os vendedores de plantas medicinais encontrados ${ }^{(16)}$ pelos pesquisadores.

Utilizou-se para coleta de dados um questionário contendo 21 questões fechadas e abertas, que foram posteriormente categorizadas, elaboradas pelos pesquisadores especialmente para esta pesquisa, com objetivo de analisar variáveis: sociodemográficas (idade - classificadas em cinco categorias: 18 a 29 anos, 30 a 39 anos, 40 a 49 anos, 50 a 59 anos, 60 anos ou mais; sexo - masculino ou feminino) e referentes às espécies vegetais de uso medicinal com indicação para tratamento de feridas (indicações, preparos e partes utilizadas). Adicionalmente, obtiveram-se informações com o raizeiro sobre: tempo de trabalho com o comércio de plantas, de onde vem o conhecimento sobre plantas medicinais, procedência das ervas, efeitos indesejados e critérios de comercialização.

A aplicação dos questionários foi realizada por dois pesquisadores devidamente treinados e de forma coordenada, em que o pesquisador efetuava a leitura e explicação do que se tratava e como deveria ser respondida cada questão. Todo o procedimento de coleta de dados teve duração de aproximadamente 15 a 20 minutos. As entrevistas aconteceram em locais reservados e em horários de menor movimentação de clientes, de forma a garantir aos entrevistados que não seriam atrapalhados em suas atividades laborais e possibilitar a coleta de informações sem interrupções frequentes.

Inicialmente, os dados foram transcritos para o Microsoft Office Excel 2010, e posteriormente importados para o software "Statistical Package for Social Science"-SPSS versão 18.0. Utilizou-se estatística descritiva (frequências absolutas e relativas) para as variáveis contínuas, e os dados referentes às plantas e aos conhecimentos dos raizeiros sobre o tratamento de feridas foram padronizados e dispostos em quadros demonstrativos. Os demais dados foram categorizados.

O presente estudo foi submetido e aprovado pelo Comitê de Ética em Pesquisa do Centro de Ensino Superior e Desenvolvimento (CEP/CESED), em conformidade com os princípios éticos dispostos na Resolução 466/12 do Conselho Nacional de Saúde ${ }^{(17)}$.

\section{RESULTADOS}

No presente estudo, foram convidados a participar da pesquisa 40 vendedores de plantas medicinais, dos quais 32 (75\%) concordaram com a entrevista. Destes, $75 \%(n=24)$ eram do sexo feminino, $50 \%(\mathrm{n}=16)$ possuíam 60 anos ou mais, $25 \%(\mathrm{n}=8)$ residiam na zona rural, e apenas $18,75 \%$ $(n=6)$ cultivavam algumas das ervas que comercializavam, comprando as demais de vendedores de outras regiões para revenda.

Segundo os raizeiros entrevistados, o tempo exercido com o comércio de plantas medicinais variou de oito meses até 25 anos de experiência na área, sendo que 87,5\% $(n=28)$ dos entrevistados trabalhavam neste ramo num tempo superior a cinco anos.

Referente à aquisição de conhecimento sobre o tratamento de feridas com plantas medicinais, os comerciantes relataram que a aprendizagem provém de fontes variadas, sendo a forma tradicional ou familiar a mais comum $(87,5 \%$; $n=28)$; seguida pelos meios de comunicação em massa: televisão, rádio, revista $(25 \% ; \mathrm{n}=8)$; médicos, rezadeiras, biólogos, professores $(18,75 \%$; $n=6)$, cursos ou oficinas não reconhecidos por órgãos fiscalizadores $(12,5 \%$; $\mathrm{n}=4)$, e outros meios não inclusos no questionário ( $43,75 \%$; $\mathrm{n}=14$ ), relacionados à sua experiência de vida na zona rural, pesquisas em trabalhos e livros, auxílio da internet e aquisição de conhecimentos através de amigos e outros vendedores.

Do total de 18 plantas recomendadas pelos raizeiros para o tratamento de feridas, a mais indicada foi o cajueiro roxo, apontada por $87,50 \%(\mathrm{n}=28)$ dos comerciantes entrevistados, seguida pelo barbatimão $(81,25 \%$; $n=26)$ e pela quixaba $(50 \% ; n=16)$.

O Quadro I demonstra os dados encontrados na presente pesquisa, confronta-os com literatura ${ }^{(18)}$, onde fica claro que a indicação das plantas medicinais não seguiu bases científicas. 
Quadro I - Plantas medicinais indicadas pelos raizeiros para o tratamento de feridas, partes utilizadas e modo de preparo de uso popular e segundo a literatura consultada. Campina Grande, PB, 2012-2013.

\begin{tabular}{|c|c|c|c|c|c|c|}
\hline \multicolumn{2}{|c|}{ Plantas medicinais indicadas } & \multicolumn{2}{|c|}{ Partes utilizadas } & \multicolumn{2}{|c|}{ Modo de uso } & \multirow{2}{*}{$\begin{array}{c}\text { Confirmação } \\
\text { Indicação* }\end{array}$} \\
\hline $\begin{array}{c}\text { Plantas } \\
\text { Indicadas }\end{array}$ & Nome científico & Raizeiros & Literatura & Raizeiros & Literatura & \\
\hline Ameixeira & Ximenia americana & $\begin{array}{c}\text { Casca e } \\
\text { Entrecasca }\end{array}$ & $\begin{array}{l}\text { Semente e } \\
\text { casca }\end{array}$ & Chá e tintura & $\begin{array}{l}\text { Decocção, macerado, } \\
\text { tintura }\end{array}$ & SIM \\
\hline Aroeira & $\begin{array}{l}\text { Myracrodruon } \\
\text { urundeuva }\end{array}$ & $\begin{array}{c}\text { Casca e } \\
\text { Entrecasca }\end{array}$ & $\begin{array}{l}\text { Casca, } \\
\text { entrecasca e } \\
\text { resina }\end{array}$ & $\begin{array}{l}\text { Chá, tintura } \\
\text { e pó }\end{array}$ & $\begin{array}{c}\text { Tintura, macerado e } \\
\text { decocção }\end{array}$ & SIM \\
\hline Babosa & Aloe vera & $\begin{array}{l}\text { Casca e } \\
\text { Folha }\end{array}$ & Folha & $\begin{array}{l}\text { Chá, tintura e } \\
\text { macerado }\end{array}$ & $\begin{array}{l}\text { Pó, infusão, extrato } \\
\text { seco e pasta da folha } \\
\text { seca }\end{array}$ & SIM \\
\hline Barbatimão & $\begin{array}{l}\text { Stryphnodendron } \\
\text { barbatiman }\end{array}$ & $\begin{array}{c}\text { Casca e } \\
\text { Entrecasca }\end{array}$ & $\begin{array}{l}\text { Casca e } \\
\text { folhas }\end{array}$ & $\begin{array}{l}\text { Chá, tintura, } \\
\text { garrafada } \\
\text { e pó }\end{array}$ & $\begin{array}{l}\text { Infusão das folhas, } \\
\text { decocção da casca e } \\
\text { macerado }\end{array}$ & SIM \\
\hline $\begin{array}{l}\text { Cajueiro } \\
\text { Roxo }\end{array}$ & $\begin{array}{l}\text { Anacardium } \\
\text { occidentale }\end{array}$ & $\begin{array}{l}\text { Raiz e } \\
\text { Casca }\end{array}$ & Entrecasca & $\begin{array}{l}\text { Chá, tintura, } \\
\text { xarope e } \\
\text { garrafada }\end{array}$ & $\begin{array}{l}\text { Macerado, decocção, } \\
\text { extrato fluido e tintura }\end{array}$ & SIM \\
\hline $\begin{array}{l}\text { Cascara } \\
\text { Sagrada }\end{array}$ & $\begin{array}{l}\text { Rhamnus } \\
\text { purshianus }\end{array}$ & Entrecasca & $\begin{array}{l}\text { Casca e } \\
\text { entrecasca }\end{array}$ & Tintura e pó & Chá e infusão & NÃO \\
\hline Confrei & $\begin{array}{l}\text { Symphytum } \\
\text { officinale }\end{array}$ & Folha & $\begin{array}{l}\text { Raízes e } \\
\text { folhas }\end{array}$ & Tintura & $\begin{array}{l}\text { Tintura, infusão, } \\
\text { cataplasma e decocção }\end{array}$ & SIM \\
\hline Copaíba & $\begin{array}{l}\text { Copaifera } \\
\text { cearenses }\end{array}$ & Óleo & Óleo e resina & Óleo & Óleo essencial e resina & SIM \\
\hline Cumarú & Dipteryx odorata & Casca & $\begin{array}{l}\text { Casca e } \\
\text { semente }\end{array}$ & Chá & $\begin{array}{l}\text { Decocção, tintura e } \\
\text { extrato fluido }\end{array}$ & NÃO \\
\hline Favela & $\begin{array}{l}\text { Cnidosculus } \\
\text { phyllacanthus }\end{array}$ & $\begin{array}{l}\text { Raiz e } \\
\text { Casca }\end{array}$ & Casca & Chá e pó & Decocção e macerado & SIM \\
\hline Ipê Roxo & $\begin{array}{l}\text { Tabebuia } \\
\text { avellanedae }\end{array}$ & Entrecasca & Casca & Chá & $\begin{array}{l}\text { Decocção, macerado, } \\
\text { extrato fluido }\end{array}$ & SIM \\
\hline João Mole & Pisonia tomentosa & Casca & Casca & Pó & Infusão & NÃO \\
\hline Juá & Zizyphus joazeiro & Casca & $\begin{array}{l}\text { Casca, Fruto } \\
\text { e Folhas }\end{array}$ & Chá & $\begin{array}{l}\text { Decocção, infusão, } \\
\text { extrato fluido e tintura }\end{array}$ & NÃO \\
\hline Jucá & Caesalpinia férrea & Casca & $\begin{array}{l}\text { Casca, raízes, } \\
\text { folha e fruto }\end{array}$ & Tintura & Infusão e extrato fluido & SIM \\
\hline $\begin{array}{l}\text { Jurema } \\
\text { Preta }\end{array}$ & $\begin{array}{l}\text { Mimosa } \\
\text { acutistipula }\end{array}$ & Entrecasca & Casca & Tintura & $\begin{array}{c}\text { Infusão, decocção e } \\
\text { tintura }\end{array}$ & NÃO \\
\hline $\begin{array}{l}\text { Quina- } \\
\text { Quina }\end{array}$ & Coutarea hexandra & Casca & $\begin{array}{l}\text { Folhas e } \\
\text { casca }\end{array}$ & Chá & $\begin{array}{l}\text { Infusão de folhas e } \\
\text { decocção de cascas }\end{array}$ & SIM \\
\hline Quixabeira & $\begin{array}{l}\text { Sideroxylon } \\
\text { obtusifolium }\end{array}$ & $\begin{array}{l}\text { Raiz e } \\
\text { Casca }\end{array}$ & Casca & $\begin{array}{l}\text { Chá, tintura e } \\
\text { garrafada }\end{array}$ & $\begin{array}{l}\text { Macerado na água, } \\
\text { decocção, tintura e } \\
\text { extrato }\end{array}$ & SIM \\
\hline Romã & Punica Granatum & $\begin{array}{l}\text { Casca, } \\
\text { Semente }\end{array}$ & $\begin{array}{l}\text { Casca do } \\
\text { caule e da } \\
\text { romã, folhas, } \\
\text { raiz, arilo } \\
\text { e polpa da } \\
\text { semente }\end{array}$ & Chá e tintura & $\begin{array}{l}\text { Macerado, extrato } \\
\text { fluido, infusão e } \\
\text { decocção }\end{array}$ & SIM \\
\hline
\end{tabular}

Fonte: Dantas, $\mathrm{IC}^{(18)}$ * Indicação para tratar feridas

\section{DISCUSSÃO}

Verificou-se, no presente estudo, que as mulheres estão mais envolvidas no trabalho com plantas medicinais que os homens, e que na maioria das vezes, o conhecimento sobre plantas provém da família. Este dado pode estar relacionado ao fato histórico das mulheres, desde o início das civilizações, serem responsáveis por cuidar da família, porém sem reconhecimento ou poder social, e que a aquisição de conhecimento geralmente era realizada pela 
aprendizagem empírica de maneiras de cuidar e preservar a saúde, transmitida em seio familiar através das gerações ${ }^{(1)}$. Essa prática, normalmente acontece em sociedades rurais ou indígenas, nas quais o aprendizado é realizado pela socialização, sem instruções mediadoras ${ }^{(19)}$.

Curiosamente, na pesquisa também realizada em Campina Grande-PB, entre 2000 e 2001, constatou-se que não há diferença significativa entre a quantidade de ervanários do sexo masculino e feminino atuantes nesta área. Essa contradição demonstra que o número de profissionais de cada gênero trabalhando nessa área é flutuante e que merece maior atenção e estudo para entender o porquê dessa disparidade ${ }^{(20)}$.

É importante destacar que boa parte dos raizeiros entrevistados na atual pesquisa não se limitou ao conhecimento repassado por seus antepassados, e citaram mais de uma fonte de aprendizado, o que demonstra a busca incessante de saberes acerca das plantas medicinais. Tal achado também foi relatado em pesquisa realizada em Vitória da Conquista-BA, onde 93\% dos herbolários obtiveram seus conhecimentos através da tradição familiar, porém apenas $7 \%$ adquiriram conhecimento teórico de outras formas (livros e internet) ${ }^{(21)}$.

No que diz respeito à idade, os resultados do presente estudo divergem dos achados anteriores, pois apenas $23,1 \%$ dos participantes possuíam 60 anos ou mais ${ }^{(21)}$.

No Brasil, devido ao seu custo elevado, $66 \%$ da população não tem acesso aos fármacos alopáticos, e $62,9 \%$ dos brasileiros frequentemente utilizam saberes populares e práticas complementares para os cuidados com a saúde, inclusive no tratamento de feridas ${ }^{(22)}$.

Entretanto, do mesmo jeito que apresentam propriedades benéficas, as plantas podem ser tóxicas e prejudiciais ao organismo humano, se utilizadas de forma inadequada ou em grandes quantidades ${ }^{(23)}$. Por esta razão, e associado ao desejo de aprender, destaca-se a necessidade de inserir estes profissionais em cursos de capacitação, na tentativa de minimizar risco à população ${ }^{(12)}$.

Com relação às plantas medicinas, os achados mais importantes no presente estudo foram referentes à Cáscara Sagrada, Cumaru, João Mole, Juazeiro e Jurema Preta. Parte dos raizeiros entrevistados indicaram essas plantas para o tratamento de feridas, contudo, nas bases de dados e literaturas consultadas a utilização delas se dá sobre outros aspectos.

A Cáscara Sagrada, Rhamnus purshianus, diferente do que recomendaram os raizeiros da presente pesquisa, é utilizada no mundo todo como laxante, seu uso contra a constipação intestinal também é muito famoso. Entre os meios de utilização, pode-se destacar o uso contra a constipação crônica, discinésia biliar, colecistite crônica, litíase biliar e meteorismo não tendo indicação para o tratamento de feridas ${ }^{(24)}$.

Contrariando o que dizem os raizeiros entrevistados no estudo em questão, a planta Cumaru, A. cearenses, não possui indicação de uso no tratamento de feridas. Esta planta possui ação expectorante, emenagoga, antiinflamatória, broncodilatadora, antirreumática, aromatizante, antiespasmódica e analgésica. Além da indicação nos casos de defluxo, sinusite, coriza, tosse, bronquite, afecções pulmonares, dores reumáticas, inflamação da garganta, asma, vitiligo, edema, perturbações digestivas, dor de barriga, cólicas intestinas e cólicas uterinas. Sendo esta planta indicada para tratamento de disfunções em vias aéreas e não em reparação tissular ${ }^{(25)}$.

A planta João Mole, Pisonia tomentosa, foi indicada, por parte dos entrevistados da atual pesquisa, por ser cicatrizante, com sua utilização na forma de pó feito da casca da planta para por sobre a ferida. Porém, esta planta possui em sua madeira um pó irritante à pele humana. Seu uso é indicado para garganta e reumatismo devendo ser utilizado como infusão da casca para gargarejo, não sendo indicação para o tratamento de feridas ${ }^{(26)}$.

O Juazeiro, Zizyphus joazeiro, possui propriedades como tônico capilar, estomacal, hipotensor, cardiotônica, digestiva, desinfetante, dentifrícia, detergente, antifebril, expectorante, antimicrobiana, antiulcerogênica, adstringente e diurética ${ }^{(27)}$. Em 2011, demonstrou-se a atividade alelopática que as sementes de Juá possuem ${ }^{(28)}$. Já em 2012, um estudo demonstrou a eficácia da escovação ecológica com o Juá no controle do biofilme dentário em crianças ${ }^{(29)}$. Apesar dos esforços e de ampla pesquisa, não foi encontrado na literatura trabalhos envolvendo o Juazeiro no tratamento de feridas.

A Jurema Preta, Mimosa hostilis, possui ação sedativa, narcótica, estupefaciente, hipnótico, anti-histérica, afrodisíaca, antiespasmódica, febrífuga, hemostático, antianêmico, anticatarral, antidiarréico e adstringente ${ }^{(30)}$. A planta é indicada nos casos de úlceras, cancro, furúnculos, erisipela, eczema, e em casos de gonorreia, não sendo indicada no tratamento de feridas de pele ${ }^{(31)}$. Estudo realizado em 2011 apontou sobre as funções alucinógenas e adstringentes da planta, contudo, os autores deixam bem claro o pouco número de estudos sobre essa espécie. Não há relatos de uso dessa planta para o tratamento de feridas na literatura consultada ${ }^{(32)}$.

Com o presente estudo foi possível constatar que a variedade de plantas medicinais comercializadas pelos raizeiros para o tratamento de feridas é extensa na cidade de Campina Grande - PB. Contudo, se encontrou limitações de tempo e recursos impossibilitando o deslocamento para investigação nas cidades adjacentes, e, consequentemente, 
o conhecimento dos profissionais liberais comerciantes de plantas medicinais não pôde ser avaliado de forma abrangente.

Dada a importância de práticas alternativas e menos dispendiosas para o restabelecimento e promoção em saúde de populações carentes, o uso de plantas medicinais pode se tornar uma estratégia integrada à atenção primária em saúde, mas para tanto, faz-se necessário um maior conhecimento do tema para as populações, profissionais liberais e de saúde.

\section{CONCLUSÃO}

Este estudo deixa em aberto a questão sobre o nível de conhecimento popular de raizeiros investigados acerca do uso de plantas medicinais no tratamento de feridas, visto que o mesmo advém de fonte familiar sem cientificidade, com imprecisa indicação terapêutica

\section{REFERÊNCIAS}

1. Badke MR, Budó MLD, Alvim NAT, Zanetti GD, Heisler EV. Saberes e práticas populares de cuidado em saúde com o uso de plantas medicinais. Texto \& Contexto Enferm. 2012;21(2):363-70.

2. Rossato AE, Chaves TRC. Fitoterapia racional: aspectos taxonômicos, agroecológicos, etnobotânicos e terapêuticos. In: Rossato AE, Pierini MM, Amaral PA, Santos RR, Citadini-Zanette V. Fitoterapia racional: aspectos taxonômicos, agroecológicos, etnobotânicos e terapêuticos. 1 ed. Florianópolis: DIOESC; 2012. p. 15-39.

3. Piresa AM, Araujo PS. Percepção de risco e conceitos sobre plantas medicinais, fitoterápicos e medicamentos alopáticos entre gestantes. Rev Baiana Saúde Pública. 2011;35(2):320-33.

4. Souza CMP, Brandão DO, Silva MSP, Palmeira AC, Simões MOS, Medeiros ACD. Utilização de plantas medicinais com atividade antimicrobiana por usuários do serviço público de saúde em Campina Grande Paraíba. Rev Bras Plantas Med. 2013;15(2):188-93.

5. Battisti C, Garlet TMB, Essi L, Horbach RK, Andrade A, Badke MR. Plantas medicinais utilizadas no município de Palmeira das Missões, RS, Brasil. Rev Bras Biociência. 2013;11(3):338-48.

6. Rosa RL, Barcelos ALV, Bampi G. Investigação do uso de plantas medicinais no tratamento de indivíduos com diabetes melito na cidade de Herval D' Oeste - SC. Rev Bras Plantas Med. 2012;14(2):306-10.
7. Firmo WCA, Menezes VJM, Passos CEC, Dias CN, Alves LPL, Dias ICL, et al. Contexto histórico, uso popular e concepção científica sobre plantas medicinais. Cad. Pesqui (São Luis). 2011;18(esp): 90-5.

8. Araujo MA, Lemos ICS, Menezes IRA, Fernandes GP, Kenrtopf MR. Uso de plantas medicinais para o tratamento de feridas. Rev. Interd. 2015;8(2):60-7.

9. Piriz MA, Lima CAB, Jardim VMR, Mesquita MK, Souza ADZ, Heck RM. Plantas medicinais no processo de cicatrização de feridas: uma revisão de literatura. Rev Bras Plantas Med. 2014;16(3):628-36.

10. Laureano A, Rodrigues AM. Cicatrização de Feridas. Rev Soc Portug Dermat Venerol. 2011;69(3):355-67.

11. Silva LL, Lopes PF, Monteiro MHDA, Macedo HW. Importância do uso de plantas medicinais nos processos de xerose, fissuras e cicatrização na diabetes mellitus. Rev Bras Plantas Med. 2015;17(4 Supl 1):827-35.

12. Santos RL, Guimaraes GP, Nobre MSC, Portela AS. Análise sobre a fitoterapia como prática integrativa no Sistema Único de Saúde. Rev Bras Plantas Med. 2011;13(4):486-91.

13. Lima PGC, Coelho-Ferreira M, Oliveira R. Plantas medicinais em feiras e mercados públicos do Distrito Florestal Sustentável da BR-163, estado do Pará, Brasil. Acta Bot Bras. 2011;25(2):422-34.

14. Antonio GD, Tesser CD, Moretti-Pires RO. Phytotherapy in primary health care. Rev Saúde Pública. 2014;48(3):541-53.

15. Lima FA, Do Bú EA, Soares MP, Araújo CRF. A fitoterapia e sua inserção no contexto da atenção básica. Rev Saúde Ciência Online [Internet]. 2015 [acesso em 2015 Ago 26];4(2):120-8. Disponível em: www:///C:/ Users/731095830/Downloads/259-485-1-SM.pdf

16. Gil AC. Como elaborar projetos de pesquisa. $5^{\text {a }}$ ed. São Paulo: Atlas; 2010.

17. Ministério da Saúde (BR). Resolução $n^{\circ}$ 466, de 12 de dezembro de 2012. Aprova as diretrizes e normas regulamentadoras de pesquisas envolvendo seres humanos [estatuto na internet]. Diário Oficial da República Federativa do Brasil, Brasília (DF), 2012 dez 12; Seção 1:137.

18. Dantas IC. O raizeiro. $22^{\mathrm{a}}$ ed. Campina Grande: EDUEPB; 2007.

19. Soares FP, Freire NM, Souza TR. Avaliação farmacognostica e da rotulagem das drogas vegetais boldo-do-chile (Peumus boldus Molina) e camomila 
(Matricaria recutita L.) comercializadas em Fortaleza, CE. Rev Bras Plantas Med. 2015;17(3):468-72.

20. Dantas IC, Guimarães FR. Perfil dos raizeiros que comercializam plantas medicinais no município de Campina Grande, PB. Rev Biologia Ciências Terra. 2006;6(1):39-44.

21. Silva PA, Faria LA, Espinheira MJCL, Mascarenhas GDM, Silva KO. O perfil dos raizeiros e a comercialização de plantas medicinais em feiras livres do município de Vitória da Conquista, Bahia. Rev Exatas Online [Internet]. 2014 [acesso 2015 Ago 26]; 5(2):8-18. Disponível em: http://www2.uesb.br/ exatasonline/images/V5N2pag8-18.pdf

22. Silva RS, Matos LSL, Araujo EC, Paixao GPN, Costa LEL, Pereira Álvaro. Práticas populares em saúde: autocuidado com feridas de usuários de plantas medicinais. Rev Enferm UERJ. 2014;22(3):389-95.

23. Carvalho APS, Conceição GM. Utilização de plantas medicinais em uma área da estratégia de saúde da família, Caxias, Maranhão. Enciclopédia Biosfera. 2015;11(21):3477-88.

24. Darroz JV, Fuso LC, Borges NM, Gomes AJPS. Utilização de fitoterápicos no tratamento de constipação intestinal. Arq Ciências Saúde UNIPAR. 2014;18(2):113-9.

25. Roque AA, Rocha RM, Loiola MIB. Uso e diversidade de plantas medicinais da Caatinga na comunidade rural de Laginhas, município de Caicó, Rio Grande do Norte (Nordeste do Brasil). Rev Bras Plantas Med. 2010;12(1):31-42.

26. Bastos B, Roge AG, Martines Y. Flora da Serra do Cipó flora nativa. Bol Bot. 2013;25(2):41-52.

27. Leite AF, Bonini G, Imparato JC, Raggio D, Menezes V, Cabral R. Avaliação da Eficácia da Escova Ecológica e do Juá no Controle de Biofilme Dentário em Crianças. Pesqui Bras Odontopediatria Clín Integr. 2012;12(3):337-43.
28. Coelho MFB, Maia SSS, Oliveira AK, Diogenes FEP. Atividade alelopática de extrato de sementes de juazeiro. Hortic Bras. 2011;29(1):108-11.

29. Leite AF, Bonini G, Imparato JC, Raggio D, Menezes V, Cabral R. Avaliação da eficácia da escova ecológica e do juá no controle de biofilme dentário em crianças. Pesqui Bras Odontopediatria Clín Integr. 2012;12(3):337-43.

30. Gardner D, Riet-Correa F, Lemos D, Welch K, Pfister J, Panter K. Teratogenic effects of Mimosa tenuiflora in a rat model and possible role of N-methyl- and N,N-dimethyltryptamine. J Agric Food Chem. 2014;62(30):7398-401.

31. Bitencourt MA, Souza LMC, Torres-Rêgo M, Fernandes JM, Silva-Júnior AA, Tambourgi DV, et al. Neutralizing effects of Mimosa tenuiflora extracts against inflammation caused by Tityus serrulatus scorpion venom. Biomed Res Int. 2014;2014:378235.

32. Souza RSO, Albuquerque UP, Monteiro JM, Amorim ELC. Jurema Preta (mimosa tenuiflora [wild.] Poir.): a review of its traditional use, phytochemistry and pharmacology. Braz Arch Biol Technol. 2008;51(5):937-47.

\section{Endereço para correspondência:}

Diogo Rodrigues Souza

Universidade Estadual da Paraíba - UEPB

Rua Baraúnas, 351 - $3^{\circ}$ Andar

Bairro: Universitário

CEP: 58.429-500 - Campina Grande - PB - Brasil

E-mail: diroso84@gmail.com 